\title{
A multi-criteria assessment of decision support systems in educational environments
}

\author{
Amjad Alowaigl ${ }^{1}$, Khalil H. A. Al-Shqeerat ${ }^{2}$, Mohammed Hadwan ${ }^{3}$ \\ 1,2 Department of Computer Science, College of Computer, Qassim University, Buraydah, Saudi Arabia \\ ${ }^{3}$ Department of Information Technology, College of Computer, Qassim University, Buraydah, Saudi Arabia \\ ${ }^{3}$ Department of computer science, College of Applied Sciences, Taiz University, Taiz, Yemen
}

\begin{tabular}{l} 
Article Info \\
Article history: \\
Received Oct 4, 2020 \\
Revised Mar 20, 2021 \\
Accepted Mar 30, 2021 \\
\hline Keywords: \\
Analytic hierarchy process \\
Decision support systems \\
Educational environment \\
Simple multi-attribute rating \\
technique
\end{tabular}

Article Info

icle history:

Received Oct 4, 2020

Revised Mar 20, 202

Keywords:

Analytic hierarchy process

Educational environmen technique

\begin{abstract}
Decision support systems (DSS) are useful business intelligence (BI) tools as they help managers in large organizations make the best out of many decisions. Decisions are based on various types of raw data, models, documents, knowledge, and past experiences. This paper examines numerous criteria of decision support systems in the educational environment. Two effective methods were discovered and applied in this research, the analytic hierarchy process (AHP) and simple multi-attribute rating technique (SMART). These methods were selected due to their abilities to deal with complex decisional environments in general and widely used in practice for the educational environment in specific. The performance of methods is compared using two datasets called xApi-Education and IPEDS datasets. The obtained results based on the measurement of space complexity showed the level of convergence and similarity between these two methods. However, the experiments show that the simple multi-attribute rating technique outperformed the analytic hierarchy process in terms of accuracy, deviation, and time complexity measurement.
\end{abstract}

This is an open access article under the CC BY-SA license.

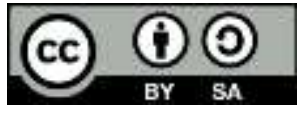

\section{Corresponding Author:}

Khalil H. A. Al-Shqeerat

Department of Computer Science

Qassim University

Buraydah, Saudi Arabia

Email: kh.alshqeerat@qu.edu.sa

\section{INTRODUCTION}

The success of decision support systems (DSS) has helped decision-makers in many business environments motivate us to investigate educational environments. This is due to the huge number of rules and regulations that need to be taken in the right decision in educational environments. DSSs are information systems that aid in business decision-making. The use of DSS has seen expanding use with relative achievements. In the competitive business industry, DSSs computerized data frameworks are utilized to assist primary leadership in big organizations. Many DSSs rely on a single parameter while in real-world most problems are multiple-parameters based. This encouraged us to investigate multiple DSS methods to solve problems in educational environments. According to [1], "DSS gives the organization a chance to analyze and process a massive amount of data and arrange them to make proper decisions. "DSSs are knowledgebased information systems that capture, handle, and analyze information that influences the decision-making performed by end-users."

The DSS classifications are essential in identifying the type of decision support system appropriately applicable to a particular organization. DSS are classified into three groups that are passive, 
active, and cooperative [2]. Passive-DSS does not recommend explicit decisions for decision-makers, but it helps during the decision making process [3]. Passive DSS is quite common in the operation of most organizations as it helps in maintaining the current position of the organization. On the other hand, ActiveDSS offers recommendations or suggestions for decision-makers. Active DSS requires the active participation of team managers to identify the gap in organizational processes and strengthen the organization's position.

Cooperative DSS is a framework designed to make decisions on behalf of decision-makers, and the choices are then refined by a decision-maker and returned for validation. It allows the decision-making process to be repeated until a satisfactory decision is reached [4]. Decision support systems come in five distinct domains [5]. These domains include; data-driven DSS, document-driven DSS, communication-driven DSS, model-driven DSS, and knowledge-driven DSS. DSS involves several domains in different industries. Figure 1 shows the DSS classification model [6]. This paper focuses on knowledge-based DSS, a learning management system as an educational decisional support system.

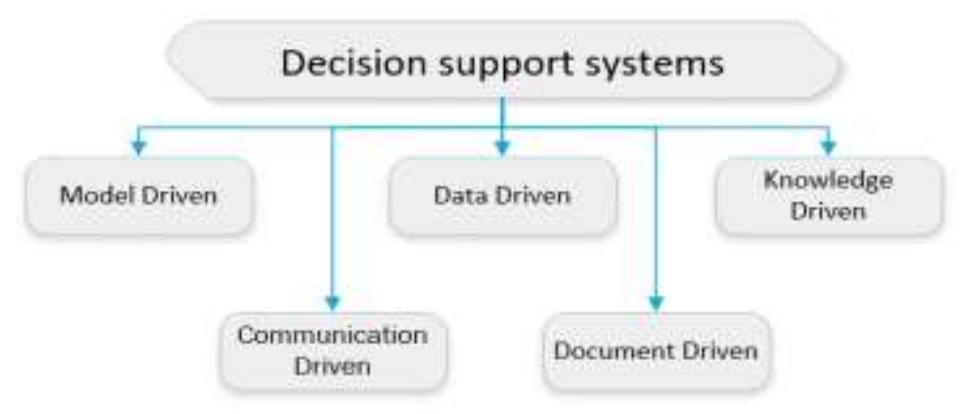

Figure 1. DSS classification model

DSS is a component of artificial intelligence (AI) where it uses intelligence techniques extensively and is referred to as intelligent decision support systems (IDSS). DSS and IDSS have primarily been used in the healthcare industry, planning, manufacturing and production finance, human resource management, environment, security, and the oil and gas industry for different purposes to solve business problems [7].

A DSS comprises of three main components [8], as shown in Figure 2:

a) A database that can also be referred to as the information distribution center. It contains structured and actual data, such as customer records, item deal history, representative calendars, or a collection of process measurements.

b) A model base which comprises of at least one model for the type of examination that the framework will carry out.

c) A user interface coordinates both within a perceived framework and gives the manager proper controls for handling information and models.

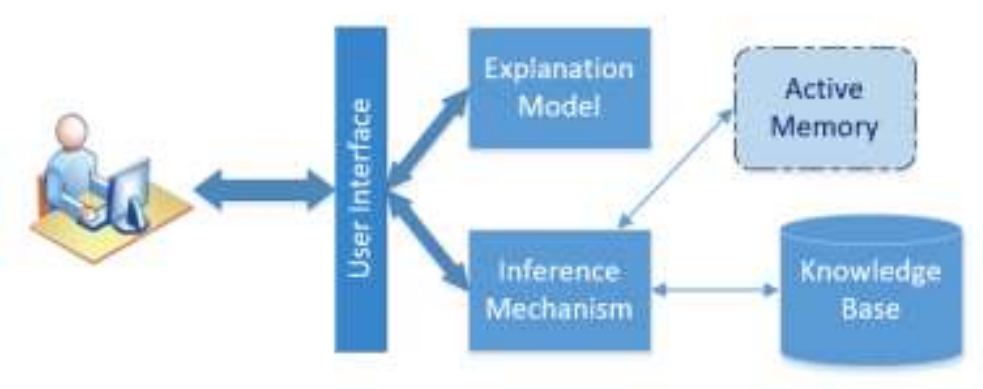

Figure 2. DSS classification model 
DSSs have been applied in many fields such as tourism, finance, planning, healthcare, medicine, and manufacturing. Moreover, it is used in planning in areas of human and natural resource management such as land-use planning, water, and energy use. DSS also assisted in strategic and planning workflow models. It eliminated problems associated with skills planning and strategic constraints in order to come up with longterm linear integer programming models to motivate personnel management [9]. In manufacturing, DSS has enabled manufacturing resource planning, especially in human engineering to meet emerging information technologies [10].

A knowledge-driven educational DSS has been significant for students and educators. Its practical application has been helpful in streamlining student administration and resource allocation in education systems [11]. In education, intelligent decision systems play a vital role in assisting educational systems to reach their decisional targets. DSS applications help in educational processes, management of learning institutions as well as coming up with a curriculum that is wholesome to the student with regard to what they are studying. Student evaluation is another aspect that uses intelligent systems. It analyses the strengths and weaknesses of students using qualitative and qualitative factors [12].

Multi-attribute decision-making methods (MADM) include elimination and choice expressing the reality (ELECTRE) method, pros and cons method, decision tree analysis, maximin and maximax techniques, conjunctive and disjunctive methods, lexicographic method, cost-benefit analysis, technique for order of preference by similarity to ideal solution (TOPSIS) method, Sensitivity tests, analytic hierarchy process (AHP) method, and simple multi-attribute rating technique (SMART) method [13]. This study focuses on AHP and SMART methods that are key to solving various problems to achieve result-oriented decisions

\subsection{Analytic hierarchy process (AHP)}

The AHP is a mathematical decision-making method coined by [14]. It is a method used to derive ration scales from comparisons that are put together. It is quite effective in dealing with complex decisions. An effective AHP needs to possess certain aspects that are specific to assist in aiding in the decision-making process. These features include; (1) Hierarchy, (2) The number of pairwise comparisons (3) Consistency (4) Collaborative voting, and (5) Sensitivity analysis.

The AHP looks at the problem in three parts, the issue in question, its alternatives, and the weights of the alternatives. It first examines the issue whose resolution is required in detail. It then looks at the possible alternatives available to solve the problem. It then evaluates the alternatives by looking at the weights of the alternatives to come up with prioritized alternative solutions. The final alternative solutions are then examined for consistency [15]. The relative weights are then got through calculation via an equation then the best solution that matches the needs is then identified as the solution to go with.

AHP is helpful in decision making. It can be used for project prioritization and selection [16]. Additionally, it allows users to record their deliberated objective as a set of guidelines for scoring projects. AHP assists decision-makers decompose critical aspects of a problem into hierarchical factors. The hierarchy then assists decision-makers to evaluate the various elements of the problem with respect to the elements that are above them in the hierarchy. However, limited by the number of evaluation criteria that are usually present in decision making as well as the usual differences in estimates made by experts as well as occasional incompatible matrices, AHP is still implementable in various fields [17]. It has been applied in various fields such as in product design, plant layout, maintenance frequency selection as well as in coming up with the choice for a logistic carrier.

\subsection{Simple multi-attribute rating technique (SMART)}

SMART is a decision-making technique that uses the multi-attribute utility theory in decision making. It is valuable in making decisions that carry value, weight, and alternatives [18]. SMART bases its framework along linear additive models to come up with decisions. It supports decision making by making simple calculating and comparing alternative values. SMART assists in goal setting as defined by its acronyms; specific, measurable, achievable, realistic, and timely. It incorporates these acronyms to help individuals focus on their effort and improve their chances of achieving the goal. The SMART model comprises of ten features; identification of the utilities, identification of the issue, identification of the alternatives, identification of the values of the individual alternatives, ranking the alternatives by importance, rating them, taking the sum of the importance weights and dividing them by the sum, measurement of the location of the alternatives, making the decision, and performing a sensitivity analysis.

SMART can be implemented in the facilitation of decision making whereby decisions need to be made from a number of alternatives. The smart technique is first implemented by identifying the alternatives that exist in relation to the decision that is to be made. Following that, the implementation of the approach then leads to the process of determining and assessing the effectiveness of the identified alternatives [19]. This way, the decision-makers will establish whether the alternatives are viable or not. Consequently, the 
next process during the implementation involves determining the optimal for the alternative selection. This is crucial since it lays down the foundation for identifying the selection criteria, which is imperative for the decision-making process.

This paper aims to apply, analyze and examine the effectiveness of DSS methods in educational environments by comparing AHP and SMART methods to analyze the best criteria and find the alternatives to aid in learning management systems decision making. The rest of the paper is organized as follows. Section 2 covers some recent literature related to DSS methods in educational environments. Section 3 elaborates on the research method. Result analysis and discussion have been presented in Section 4. Section 5 introduces the conclusion and future work.

\section{LITERATURE REVIEW}

There is significant interest from researchers in discovering the uses of DSS in various fields, especially in educational settings. Sethi et al. in [20] investigated how to solve faculty planning and staffing issues, curricula, admission and enrolment, and general learning processes. One of the first areas of DSS applications would be to make legitimate academic logical tools to collect, arrange, and evaluate data and information for capable decision-making. After that, a couple of decision-making issues in education are taken, such as planning decisions for the guidelines of study and establishing curricula, students' research and teaching, general administration, and performance evaluation. Thus, the DSS model in higher institutions encompasses students, research, and education.

In the academic planning sector, many data analysis for large volumes of data is done for multiple systems. This implies the academic workload that has to be managed. DSS is needed to adequately manage and support undergraduate educational frameworks such as colleges, degrees, courses, admission policies, and teaching workload [21]. Implementing the system as "on the fly" decision support facility by the university policymakers enables more efficient academic management.

Khalid A. Fakieh in [22] investigates the use of DSS to support higher education systems. Modules of Students, research, and teaching are introduced. In [23], researchers investigate decision support for university enrollment management. The researcher introduced a system that was able to help in decision making for enrolling students. Intelligent decision systems play a vital role in assisting educational systems to achieve their critical goals. DSS applications in education are referred to as EDSS. Intelligence systems provide the advantage of passing through large volumes of academic data quickly and accurately. In [24], authors have reviewed the human and structural characteristics inherent in DSS systems that make them usable in educational research and development. The authors argue that evaluating critical aspects can improve the quality of decisions through an appropriate and comprehensive analysis of data according to specific processes and available resources.

In agreement, Russell and Norvig suggest that fine-tuned and intelligent decisions can be deduced from the use of DSS [25]. It has become a growing important aspect of higher education development. For this to happen, it is necessary to gather all the conditions necessary. For instance, [26] indicates that the "intelligent tutoring system (ITS)" demonstrates the application of the intelligent decision support system in education. For starters, the intelligent tutoring system is a software application that expects to give prompt and tweaked guidance or criticism to learners, typically without impedance from a human educator. ITSs have the general expectation to encourage learning in a suggestive and proficient path by utilizing an assorted variety of figuring advancements. There is a lot of examples of its being utilized in both authority training and expert circumstances in which they have affirmed their capacities and limits. There is a solid relationship between wise mentoring, psychological learning hypotheses, and structure. This way, the intelligent DSS intends to take care of the issue of over-reliance of understudies over educators for prevalence training. It expects to offer access to high-class training to each understudy, therefore improving the entire instructive framework. This research investigates problems in educational systems and attempts to bridge the existing gap. There is limited research applied to specific decision systems that look into student admission and its relationship to student's course selection, enrolment, and performance. Available research focuses on general educational components of teaching, curriculum, and examination systems without concentrating on the best effective DSS method.

\section{RESEARCH METHOD}

In the challenges of multi-criteria methods, the decision-making process depends on choosing the decision-maker based on all the criteria. Traditionally, the problem is approached by primarily categorizing options using the multi-attribute utility function, so that the decisions are made as per the utility functions of different criteria. However, Khair in [6] states that it is not critical to define each utility function for all the 
criteria related to the problem since there is a selection conflict when using multiple criteria, as an individual would be willing to lose in one criterion may help win through another criterion or vice versa. This interchange affects the effectiveness of the multi-criteria utility that a decision-maker may obtain when all criteria are considered together in a multi-criteria decision problem. This section describes the research process followed. It provides information and data obtained using the SMART and AHP methods used in this study and their justification. The data collection process and result presentation are also highlighted.

\subsection{Dataset definition}

The datasets used in this study involves xApi-Education-dataset and IPEDS (Integrated postsecondary education dataset) dataset. The xAPI-Education dataset is an educational dataset collected from an e-Learning management system called Kalboard 360 using experience API web service (xAPI). xAPI is a tool to track the learner activity that enables the user to monitor learning progress [27]. It helps the learning activity providers to determine learners, activities, and objects that describe a learning experience.

The dataset consists of 480 student records and 16 features. The features are classified into three major categories: (1) Demographic features such as gender and nationality. (2) Academic background features such as educational stage, grade level, and section. (3) Behavioral characteristics include raised hand-on class, opening resources, answering surveys by parents, and school satisfaction.

The dataset involves 305 males and 175 females who are from different nationalities as follows. The are 179 students are from Kuwait, 172 students are from Jordan, 28 students from Palestine, 22 students are from Iraq, 17 students from Lebanon, 12 students from Tunis, 11 students from Saudi Arabia, 9 students from Egypt, 7 students from Syria, 6 students from USA, Iran, and Libya, 4 students from Morocco and one student from Venezuela. The dataset is collected through two academic semesters: 245 student records are collected during the first semester of the first year, and 235 student records are collected during the second semester of the first year. It also includes the school attendance feature, such as the students are classified into two categories based on their absence days: 191 students exceed 7 absence days and 289 students their absence days under seven. The students are classified into three numerical intervals based on their total grade/mark; low-level interval (0 to 69), middle-level interval (70 to 89), High-Level interval (90-100).

Furthermore, the dataset includes a new category of features; this feature is parent participation in the educational process. Parent participation features have two sub-features: Parent Answering Survey and Parent School Satisfaction. 270 parents answered the survey, and 210 are not; 292 of the parents are satisfied with the school, and 188 are not. Table 1 shows the critical attributes of xApi-Education-dataset.

Table 1. Key attributes of xApi-education-dataset

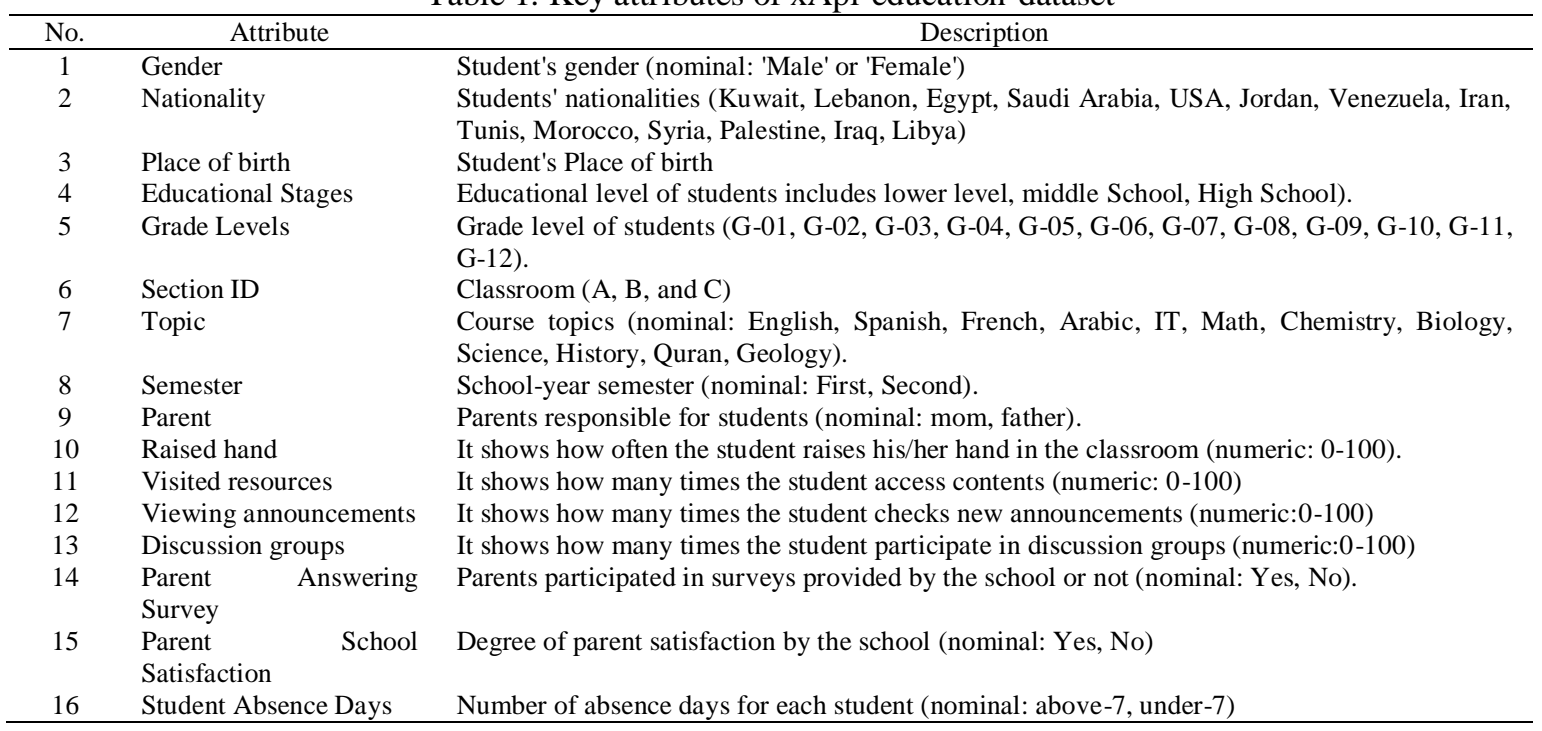

The second dataset used in this study is from the integrated postsecondary education data system (IPEDS). It was established to sort information from all postsecondary institutions in the USA [28]. Statistics produced from this data set allowed the national centre for education statistics (NCES) to describe the size of one of the nation's largest enterprises (postsecondary education) in terms of students enrolled, degrees, and other awards earned. Students have a challenge deciding which colleges they should apply to, i.e., the colleges that provide the best performance at a minimal cost given their profiles. Not only the performance in 
an examination such as SAT and ACT determine the student profile, but also other data points such as their ethnicity, immigration status, gender, and so forth.

An integrated postsecondary education dataset is presented at the aggregate level of higher education institutions despite its lack of student-level data. According to the US Department of Education, "Institutions provide information through twelve interrelated survey components collected in three periods"[29]. Table 2 shows the key attributes of IPEDS distributed over specific three educational periods.

Table 2. Main attributes used in IPEDS-dataset

\begin{tabular}{cl}
\hline Period & \multicolumn{1}{c}{ Attribute } \\
\hline Fall & Type of institution. \\
& Tuition fee estimates \\
& How many completers are in each program \\
& Degrees/certificates awarded based on the programs \\
& 12-month enrollment \\
Winter & Number of admissions \\
& Graduation rates \\
& Students performance between 6-8 years \\
& How many students received monetary help, type of aid? \\
& Average service fees \\
Spring & All census enrollment counts \\
& Enrolled distance learners \\
& Retention rates \\
& Institution expenses \\
& Staff population and their designation \\
& Amount spent on staff salary \\
& Academic libraries
\end{tabular}

\subsection{Data pre-processing phase}

This stage is an essential part of any dataset learning process. In this stage, the data is scanned for problems like missing data values, and out of range values. The first step in this process is to check for any missing values, the dataset is well prepared, or this step has been skipped, and only blank entries are checked. Since the collected data comprises many categorical data, it must be converted into numbers to enable predictive models to understand better and handle them.

The IPEDS dataset is highly erroneous. To address errors, the following procedures must be applied during the pre-processing data phase; defining minimum features, dropping missing target values, dealing with outliers, assigning missing values, measuring and normalizing data, segmenting data for validation, and finally, independent component analysis (ICA) is used to reduce the feature space.

\subsection{Data selection}

The data selection process was simple by using the entire dataset with non-blank fields. Blank fields are viewed as potentially interfering with random data that may distort the results of clustering or classification. Selecting from a random set of data is not easy since there is a wide gap that separates the similarity metrics for the data components. After filtering, there is no need for any other selection criteria because the size of the final data becomes sufficient for clustering.

The process of building the selection algorithm takes two steps. The first one is data and patterns training, which involves a classification algorithm built on the training data and recording all dataset attributes. The selection criteria are decided at this stage. The Actual Classification is the second step. It involves checking the accuracy of the algorithm before using it to classify new data. The classification labels presented in this study are in the form of distinct values that describe data.

The choice of the appropriate selection algorithm for any dataset is an intricate issue and fundamental challenge. In this study, we demonstrate the development of a meta-analysis-oriented structure to enhance the decision-making in selecting classification algorithms taking into account the dataset properties. Focusing on AHP, the result of the comparative analysis is placed in the decision matrices before running the implementation of the python code. After all, decisions have been made; criteria and alternatives priority must be calculated.

Figure 3 and Figure 4 show the main classification steps using SMART and AHP algorithms, respectively. SMART is the algorithm implementing the multiple criteria proposed in this study, while AHP is the previous algorithm with a single decision-making parameter. A decision tree classifier was used for the 
AHP because it shows all the possible alternative decisions that can be made and help to determine the probability of making each alternative decision.

\subsection{Assessment criteria}

Algorithm evaluation is a key component of determining its efficiency in the problem-solving process. Therefore, we have applied the following criteria to an educational environment to determine the accuracy and the performance of AHP and SMART algorithms [30].

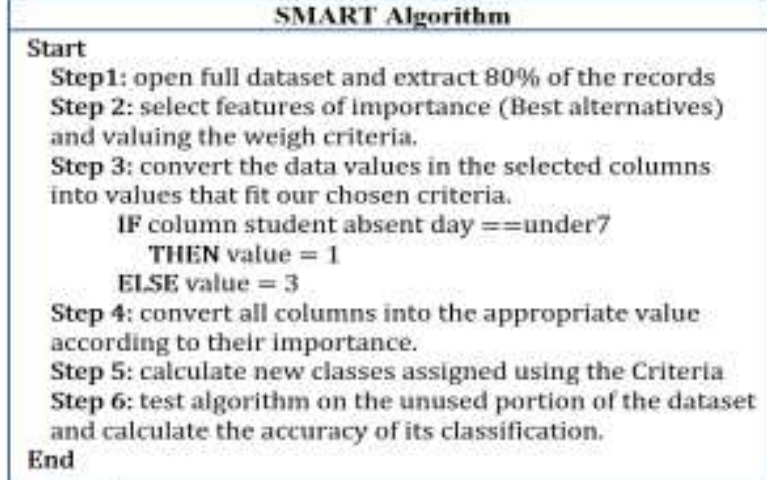

Step 1: open full dataset and extract $80 \%$ of the records Step 2: select features of importance (Best alternatives) and valuing the weigh criteria,

Step 3: convert the data values in the selected columns into values that fit our chosen criteria.

IF column student absent day $==$ under 7 THEN value $=1$

EISE value $=3$

Step 4: convert all columns into the appropriate value according to their importance.

Step 5: calculate new classes assigned using the Criteria Step 6: test algorithm on the unused portion of the dataset End

Figure 3. Dataset classification using SMART algorithm

\begin{tabular}{|l|}
\hline \multicolumn{1}{|c|}{ AHP Algorithm } \\
\hline Start \\
Step 1: open 80\% of the dataset \\
Step 2: clean dataset \\
If (field is empty) \\
delete row \\
Else \\
select Row \\
Step 3: feed data into decision tree classifier to train it \\
Step 4: use trained classifier on the rest of the data \\
Step 5: return a list of data features and their percentage \\
contribution to final decision \\
Step 6: use the list to make a classification \\
End
\end{tabular}

Figure 4. Dataset classification using AHP algorithm

\subsubsection{Accuracy}

Accuracy refers to how often an algorithm classifies a data point correctly. In an educational environment, it is significant to determine the number of the correctly predicted points out of all data points taken by the algorithm as input in order to ensure that the algorithm will positively contribute to the decisionmaking process. To illustrate the accuracy in both AHP and SMART algorithms, 100 iterations of algorithms run using different decision tree settings. Each iteration is made with the decision-maker (decision Tree algorithm) on the same setting for each iteration. During this stage, the weight to use on each data column and normalized were decided. The weights are the percentage obtained for each feature. The decision tree classifier algorithm has several stages as follows:

Stage 1: Identify the persons making the decision(s).

Stage 2: Find the issues. The utility relies on the decision extent and intent.

Stage 3: Find alternatives. It determines the outcome of certain actions.

Stage 4: Find the criteria. Limiting the value sizes is significant. It can be achieved by re-establishing or merging standards and avoiding lowly rated criteria.

Stage 5: Allocate values for individual criterion. The stage is straightforward for decisions made by one person. Grading ranks is a decision-making activity that is simpler than weights.

Stage 6: Find out the weight of each criterion: A value of 100 would be given to the most significant dimension when using a linear scale ranging from 0-100. A number that is of adequate value is allocated to the most valuable in the dimension. The method proceeds, with implied ratios reviewed as every new decision, is done. Because it involves an increasing number of comparisons, the number of measurements needs to be limited quite practically.

Stage 7: Calculate a weighted average of the values assigned to each alternative:

Stage 8: Determine a temporary decision

Stage 9: Do the analysis of sensitivity.

\subsubsection{Time and space complexity}

Enhancement of an algorithm's complexity can significantly increase its efficiency. The improved algorithm normally outperforms the original algorithm. Therefore, to measure the performance of AHP and SMART algorithms the time complexity and the space complexity of both algorithms have been computed. Time complexity can be measured by selecting the number of operations executed. Space complexity is measured by determining the memory used to perform the algorithm operations. Big O notation was used to classify algorithms' complexity according to their time and space requirements for input size $(n)$. Big $\mathrm{O}$ notation could easily show which algorithm was more complicated. 
AHP is used to obtain weights that should be used for datasets, and since it is only dependent on the number of features used, it may follow that regardless of the size of the dataset the process always takes a constant time. Therefore, in computing time complexity the constant time $(C)$ is added to the dataset size $(n)$ [31]. The classification setting is also a common method hence its time complexity is considered. Therefore, the time complexity of the AHP method can be computed using the following formula,

$$
T_{A H P}=2 n^{2}+10 n+C
$$

In General, the time complexity of the original AHP method is calculated in $O\left(\min \left\{m n^{2}, m^{2} n,\right\}\right)$ time [32], where $m$ is the amount of candidates and $n$ is the selection criteria. AHP also has a rank reversal issue when selected criteria or candidates are inserted or removed from the dataset. Rank consistency in AHP is dependent on the pairwise comparison matrix and its size. Whereas the time complexity of the SMART algorithm is also $O\left(n^{2}\right)$ according to (2),

$$
T_{S M A R T}=n^{2}+10 n+2 C
$$

On the other hand, space complexity is the amount of memory space required by decision support algorithms to solve the problem and produce results. In the AHP_weighted_dataset, independent of the dataset size, the space complexity is continuously equal to constant time. Therefore, the space complexity of the AHP method without classification will be equivalent to $+2 n$, which is $O(n)$. It means that if the input is increased, the requirement of memory also increases linearly. In the case of the SMART method, two arrays were created which are independent of dataset size but depend on the number of features a dataset has which most of the time is constant. Therefore, the space complexity for SMART is $C+4 n$ which is also $O(n)$.

\section{RESULTS ANALYSIS AND DISCUSSION}

The classification steps have been implemented in python programming language using windows 10 computer. The computer had 4GB RAM, Core i5 processor with a $3.5 \mathrm{GHz}$ processor. The obtained results and the available datasets will be used to make decisions on the appropriate institutions. The decision made from the selection of the institution was based on the class in which AHP and SMART algorithms classify a student. According to the analysis of the IPEDS dataset, it was evident that high performance in the ACTs and SATs exams lead to a higher rate of admissions. Besides, students classified as high performers in the xAPI-EDU dataset tended to perform better in ACTs and SATs exams. The student admission rate is based on the classification results from AHP and SMART algorithms, and the classes a student was classified according to the classification algorithm.

AHP algorithm utilizing two main parameters, random state, and max depth. These parameters judge the utility of the data features based on the comparison criteria to give AHP algorithm weights to the dataset. To test the proposed algorithms, 100 runs were carried out to identify the best results. It is noticed that the results were so close; that is why we just recorded the first ten runs only. Table 3 compares the first 10 iterations for SMART and AHP. The results show that SMART performs better than the AHP algorithm.

Table 3. Accuracy comparison against iterations

\begin{tabular}{ccccccccccc}
\hline \multirow{2}{*}{ Accuracy } & \multicolumn{10}{c}{} \\
& 1 & 2 & 3 & 4 & 5 & 6 & 7 & 8 & 9 \\
\hline SMART & 65.493 & 69.4444 & $\mathbf{7 5 . 7 5 7 6}$ & 67.284 & 75.3333 & 66.4122 & 71.3333 & 69.1781 & 69.2308 & 65.3333 \\
AHP & $\mathbf{6 8 . 2 8 9}$ & 63.8897 & 66.8874 & 59.4771 & 59.3333 & 55.102 & 62.1429 & 59.3985 & 65.3595 & 51.5625 \\
\hline
\end{tabular}

The average accuracy for SMART was 69.48, while 61.14 for AHP. The best results were 75.75 obtained by SMART while 68.28 for AHP. The significance of results is since these results are the first obtained results using SMART and AHP to solve IPEDS. This will help to open up the door for the researchers to investigate the educational datasets in general and IPEDS in specific in more detail and compare the future algorithms with SMART and AHP. In Figure 5, a visualization of the 100 runs is demonstrated for SMART and AHP algorithms. This visualization shows that the results are very closed and consistent for each algorithm. The figure shows clearly that the SMART algorithm performs better than AHP as the blue line represents SMART is drowned above the yellow line of AHP in all iterations. 


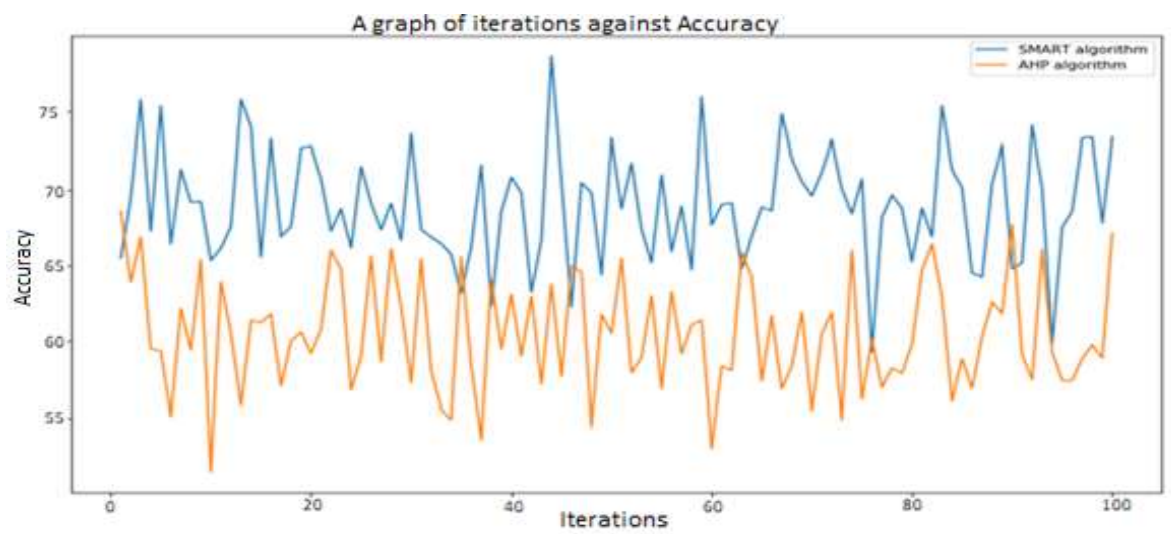

Figure 5. A graph of both AHP and SMART against accuracy

To illustrate AHP, 100 iterations of the algorithm were run using different decision tree settings and the results for the accuracy were then saved into a CSV file. The results show that the accuracy of the AHP algorithm lies between 50 and 70 even as the number of iterations approaches 100. In SMART, 100 iterations were run while changing weights of clustering criteria, and then a CSV file that contains the number of iterations against the corresponding degree of accuracy was created. The accuracy results for the SMART algorithm spread from 59 to 80, therefore depicting a high accuracy measurement than the AHP within the same number of iterations. Figure 6 compares the time complexity of both SMART and AHP with no classification. SMART outperforms AHP when it comes to scaling. The orange line represents SMART and has lower time complexity than the blue line representing AHP, which shows that it is far from $O(n)$, and it continually increases as the input grows.

On the other hand, Figure 7 shows an extreme increase in the SMART algorithm (blue line) time complexity while AHP (Orange line) remains constant without classification when we include other classification methods during Time Complexity of AHP and SMART algorithms calculations. We can infer that with an increase in the number of inputs, the time complexity increases following a quadratic relationship. A time complexity graph of the two methods plotted in the same graph is displayed to show how well both methods scaled comparing the yields of $O\left(n^{2}\right)$. Considering the classifiers, time complexity $O\left(n^{2}\right)$ is far from the constant inputs $O(n)$.

The deviation was evaluated to see how applying the calculated weights affected the datasets. The mean and standard deviation of the score (which is the total of the dataset rows after using the values) was calculated and compared for the AHP algorithm. Figure 8 shows the deviation score output for AHP and SMART methods.

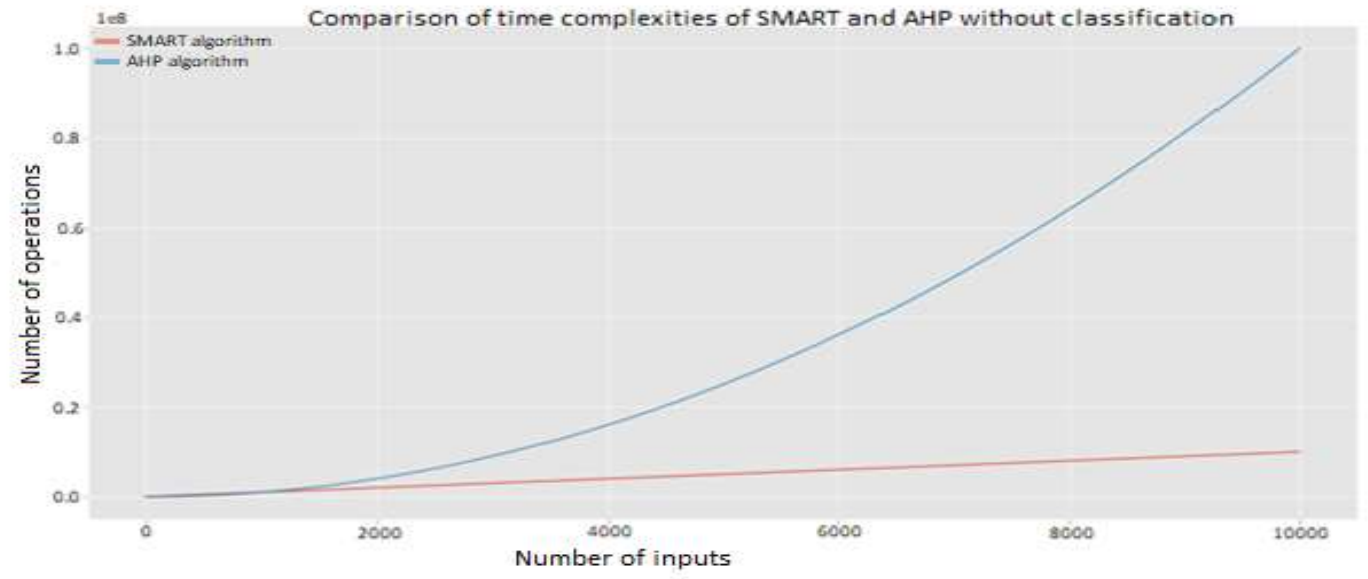

Figure 6. Time complexity without classifications 


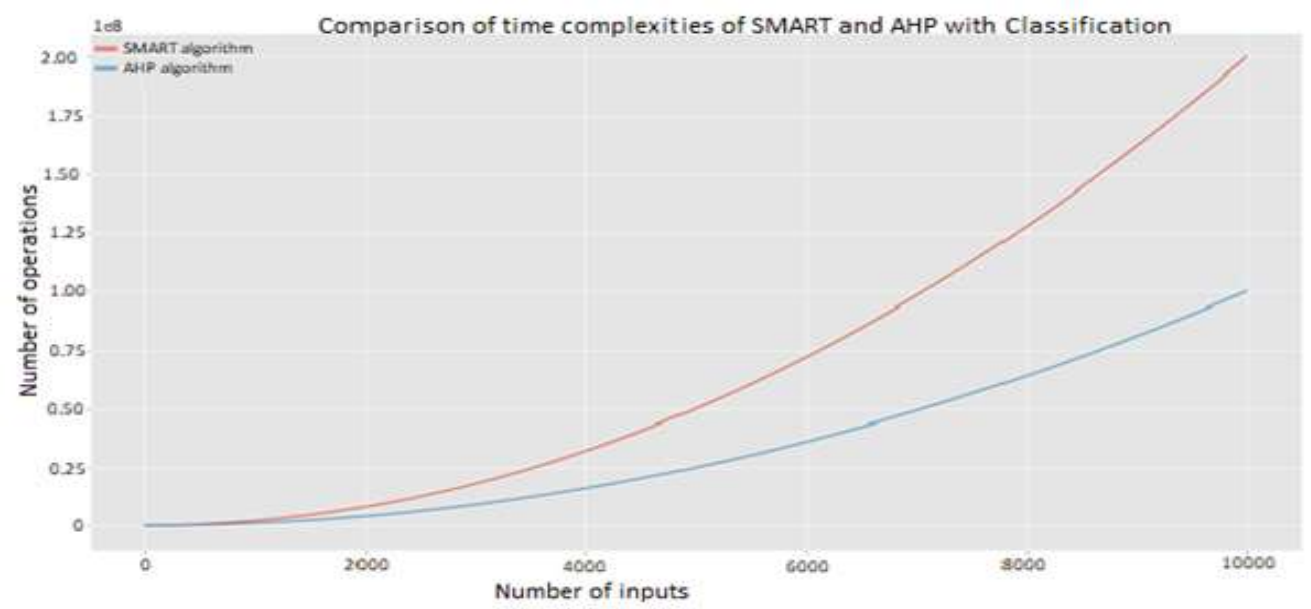

Figure 7. Time complexity with classifications

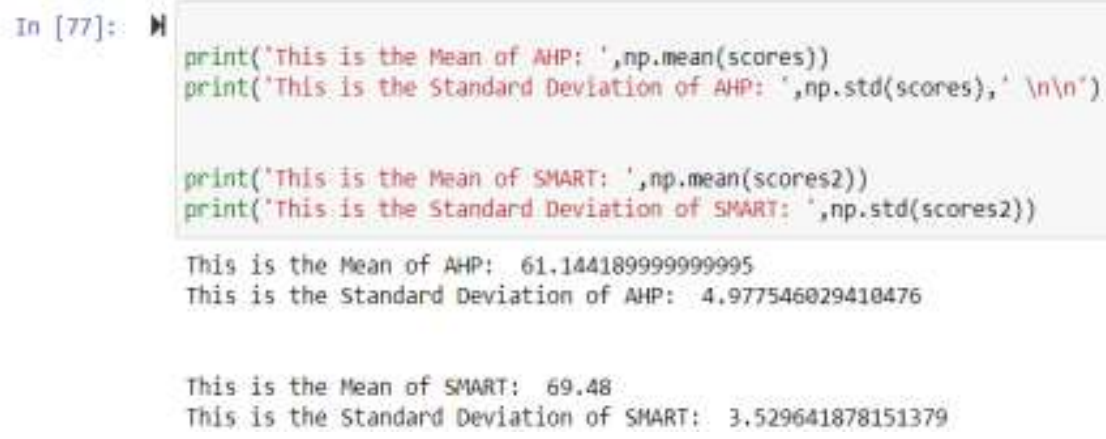

Figure 8. Deviation score output for AHP and SMART methods

Figure 9 shows the combined histogram and density plot for AHP deviation scores, while Figure 10 shows histograms and density plots that illustrate these changes. The histogram allows us to visualize the frequency distribution of the deviation. While the density plot is a smooth continuous curve estimated from the score data to enable us to visualize the deviation. After determining the accuracy, performance, and deviation of AHP and SMART algorithms, it was found that the SMART algorithm recorded a consistently high accuracy value (up to $80 \%$ ) for 100 iterations, as compared to AHP with $70 \%$ for the same number of iterations. Similarly, SMART outperforms AHP when it comes to performance. The AHP algorithm deviates further from its mean than the SMART algorithm. Therefore, making the SMART algorithm better than AHP when the Deviation is observed. Table 4 summarizes the information obtained from the study. It also shows the various criteria studied for each method.

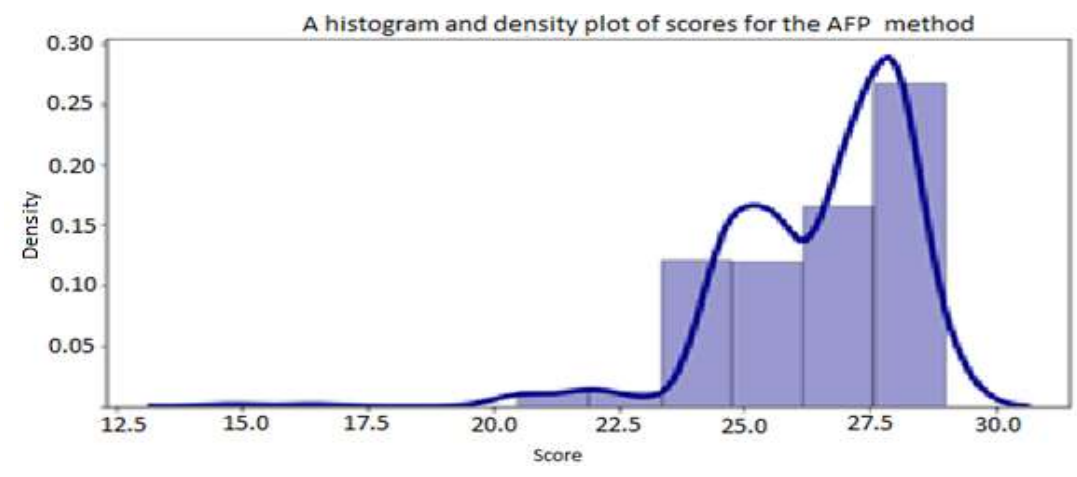

Figure 9. AHP method combined histogram and density plot for deviation 


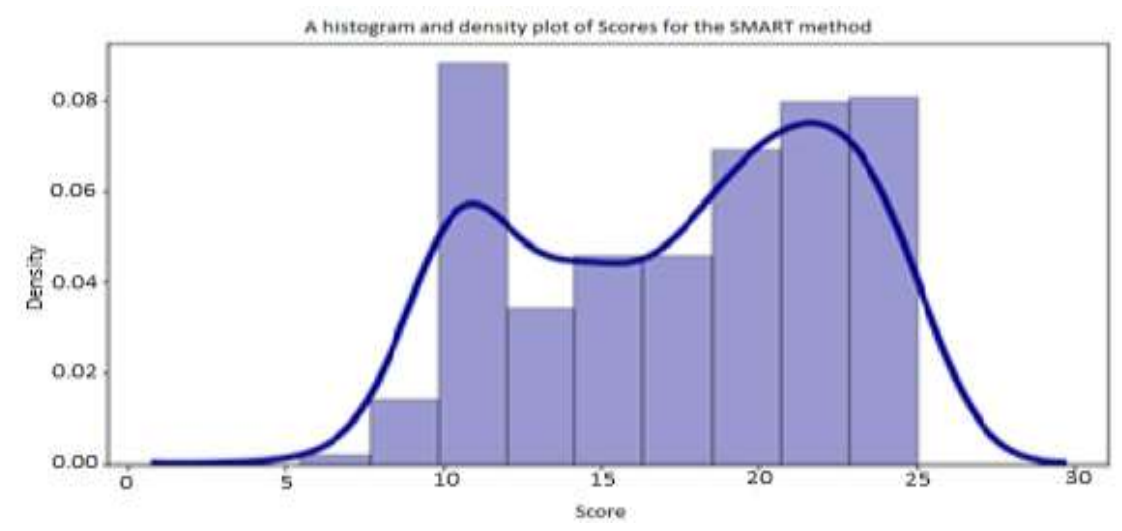

Figure 10. A combined histogram and density plot of scores for the SMART algorithm

Table 4. Results summarization

\begin{tabular}{llrl}
\hline Criteria & \multicolumn{1}{c}{ Method } & Result \\
\hline Average Accuracy & SMART & $69.48 \%$ & \\
& AHP & $61.14 \%$ & \\
Best Accuracy & SMART & $75.75 \%$ & \\
& AHP & $68.28 \%$ & \\
\multirow{2}{*}{ Worst Accuracy } & SMART & $65.33 \%$ & \\
\multirow{2}{*}{ Deviation } & AHP & $59.47 \%$ & \\
& SMART & 3.5296 & \\
Space Complexity & AHP & 4.977 & \\
& SMART & $\mathrm{O}(\mathrm{n})$ & \\
\multirow{2}{*}{ Time Complexity } & AHP & $\mathrm{O}(\mathrm{n})$ & $\mathrm{O}\left(\mathrm{n}^{2}\right)$ \\
& SMART & $n^{2}+10 n+2 C$ & $\mathrm{O}\left(\mathrm{n}^{2}\right)$ \\
\hline
\end{tabular}

\section{CONCLUSION AND FUTURE WORK}

This paper addressed the comparison of decision support methods using a multi-criteria approach for educational environments. Two methods, namely AHP and SMART, were successfully implemented and analyzed using two educational datasets. Based on obtained results, the SMART method produced accurate results up to $75.75 \%$ when datasets are weighted while $68.28 \%$ for AHP. For time complexity, SMART followed a linear computational time while AHP followed a quadric computational time. This implies that the SMART method requires less computational time for the decision making process compared with AHP. Furthermore, SMARTs' standard deviation was lower than AHP, making it more reliable and consistent. In conclusion, the SMART method performed better due to its high accuracy and reduced computational time, making it simple and easy to use in decision-making processes for the educational environment. This paper contributes to educational decision making by identifying the SMART method as an effective method to make better decisions in environmental education. In addition, to specify multiple criteria that can be used to support decisions in the educational environment. For suggested future work, researchers should investigate the hybridization between AHP and SMART methods with other methods in order to have reliable methods that can tackle complex problems. This is due to its ability to join subjective and objective criteria critical to using utilization functions. It's also flexible in dealing with an increased variety of complex data and changing times.

\section{REFERENCES}

[1] B. L. Golden, E. A. Wasil, and D. E. Levy, "Applications of the Analytic Hierarchy Process," in The Analytic Hierarchy Process, Springer Berlin Heidelberg, 1989, pp. 37-58.

[2] N. Subramanian and R. Ramanathan, "A review of applications of Analytic Hierarchy Process in operations management," Int. J. Prod. Econ., vol. 138, no. 2, pp. 215-241, Aug. 2012, doi: 10.1016/j.ijpe.2012.03.036.

[3] M. Gunduz and O. H. Khan, "Effective framework for change order management using analytical hierarchy process (AHP)," 2018. [Online]. Available: http://dergipark.gov.tr/gujs.

[4] A. Ishizaka and A. Labib, "Review of the main developments in the analytic hierarchy process," 2011. doi: 10.1016/j.eswa.2011.04.143. 
[5] R. Mohemad, A. R. Hamdan, Z. A. Othman, and N. M. M. Noor, "Decision Support Systems (DSS) in Construction Tendering Processes," IJCSI International Journal of Computer Science Issues, vol. 7, no. 1, Apr 2010.

[6] Khair, K. G. E. M. (2018). Creating A Python Toolbox to Implement The Analytical Hierarchy Process Method In ARCGIS (Doctoral Dissertation).

[7] F. Provost and T. Fawcett, "Data Science and its Relationship to Big Data and Data-Driven Decision Making," Big Data, vol. 1, no. 1, pp. 51-59, Mar. 2013, doi: 10.1089/big.2013.1508.

[8] I. H. Witten, E. Frank \& M. A. Hall, "Data Mining: Practical machine learning tools and techniques, 3rd Edition". Morgan Kaufmann, San Francisco. p. 197, 2011.

[9] A. Ciancimino, I. Lari, F. Nicolo, and M. Lucertini, "A Decision Support System for Human Resource Management: Strategic and Tactical Planning Flow Network Models," IFAC Proc. Vol., vol. 25, no. 7, pp. 421-427, May 1992, doi: 10.1016/s1474-6670(17)52403-9.

[10] R. S. Erst, "Decision Support Systems in Manufacturing: A New Approach to MRP," IFAC Proc. Vol., vol. 15, no. 8, pp. 163-167, Oct. 1982, doi: 10.1016/S1474-6670(17)62789-7.

[11] V. T. N. Chau and N. H. Phung, "A knowledge-driven educational decision support system," IEEE, 2012, doi: 10.1109/rivf.2012.6169819.

[12] E. Mallach, "Decision support and data warehouse systems," Irvin/McGraw-Hill, 2000.

[13] W. Bhuasiri, O. Xaymoungkhoun, H. Zo, J. J. Rho, and A. P. Ciganek, "Critical success factors for e-learning in developing countries: A comparative analysis between ICT experts and faculty," Comput. Educ., vol. 58, no. 2, pp. 843-855, Feb 2012, doi: 10.1016/j.compedu.2011.10.010

[14] E. Turban, R. Sharda, D. D.-G. Scholar, and U. 2010, "Decision Support and Business Intelligence Systems (required)," Academia.Edu, vol. 201330, pp. 1-11, 2013.

[15] R. Risawandi and R. Rahim, "Study of the Simple Multi-Attribute Rating Technique For Decision Support," Int. J. Sci. Res. Sci. Technol., vol. 2, no. 6, pp. 491-494, 2016.

[16] J. G. Dolan, "Shared decision-making - transferring research into practice: The Analytic Hierarchy Process (AHP)," Patient Educ. Couns., vol. 73, no. 3, pp. 418-425, Dec 2008, doi: 10.1016/j.pec.2008.07.032

[17] S. H. and Q. Song, "Motivational Framework: Insights into Decision Support System Use and Decision Performance," in Decision Support Systems, InTech, 2010, doi: 10.5772/39462

[18] D. Siregar, D. Arisandi, A. Usman, D. Irwan, and R. Rahim, "Research of Simple Multi-Attribute Rating Technique for Decision Support," J. Phys. Conf. Ser., vol. 930, no. 1, p. 12015, 2017, doi: 10.1088/17426596/930/1/012015

[19] Y. M. Huang, P. S. Chiu, T. C. Liu, and T. S. Chen, "The design and implementation of a meaningful learningbased evaluation method for ubiquitous learning," Comput. Educ., vol. 57, no. 4, pp. 2291-2302, Dec 2011, doi: 10.1016/j.compedu.2011.05.023

[20] A. Sethi, J. P. Saini, M. Bisht, and W. Delhi, "ISSN : 2249-0868 Foundation of Computer Science FCS," 2013. [Online]. Available: www.ijais.org.

[21] S. Mansmann and M. H. Scholl, "Decision support system for managing educational capacity utilization," IEEE Trans. Educ., vol. 50, no. 2, pp. 143-150, May 2007, doi: 10.1109/TE.2007.893175

[22] K. A. Fakeeh, "Decision Support Systems (DSS) in Higher Education System," International Journal of Applied Information Systems (IJAIS), vol. 9, no. 2, pp. 42-40, 2013.

[23] E. N. Maltz, K. E. Murphy, and M. L. Hand. "Decision support for university enrollment management: Implementation and experience". Decis. Support Syst., vol. 44, no. 1, pp. 106-123, Nov. 2007. doi: doi.org/10.1016/j.dss.2007.03.008

[24] L. Sumaryanti, T. K. Rahayu, A. Prayitno, and Salju, "Comparison study of SMART and AHP method for paddy fertilizer recommendation in decision support system," IOP Conf. Ser. Earth Environ. Sci., vol. 343, no. 1, 2019, doi: 10.1088/1755-1315/343/1/012207

[25] Russell, S. J., and P. Norvig, Artificial Intelligence: A Modern Approach, 2nd ed., Upper Saddle River, NJ: Prentice-Hall, 2003.

[26] de Carvalho, S.D., de Melo, F.R., Flôres, E.L. et al. "Intelligent tutoring system using expert knowledge and Kohonen maps with automated training." Neural Comput \& Applic, vol. 32, no. 23, 2020, doi: 10.1007/s00521020-04767-0

[27] E. A. Amrieh, T. Hamtini, and I. Aljarah, "Preprocessing and analyzing educational data set using X-API for improving student's performance," 2015 IEEE Jordan Conference on Applied Electrical Engineering and Computing Technologies (AEECT), Dec. 2015, doi: 10.1109/AEECT.2015.7360581

[28] NCES, "The Integrated Postsecondary Education Data System," National centre for education statistic.

[29] U.S. Department of Education, National Center for Education Statistics, Integrated Postsecondary Education Data System (IPEDS), [2016], Retrieved from https://nces.ed.gov/ipeds/Content/files/2017047_web.pdf

[30] Nazeer, KA Abdul, and M. P. Sebastian. "Improving the Accuracy and Efficiency of the k-means Clustering Algorithm." Proceedings of the world congress on engineering. Vol. 1. London: Association of Engineers, 2009.

[31] S. Devi, K. Selvam, and S. Rajagopalan, "An abstract to calculate big O factors of time and space complexity of machine code," International Conference on Sustainable Energy and Intelligent Systems (SEISCON 2011), 2011.

[32] R. K. Dewi, B. T. Hanggara, and A. Pinandito, "A Comparison Between AHP and Hybrid AHP for Mobile Based Culinary Recommendation System”. International Journal of Interactive Mobile Technologies (iJIM), vol. 12, no 1, pp. 133-140, 2018. 